\title{
A Report on Treatment of Chronic Obstructive Pulmonary Disease with Electroacupuncture Combined with Traditional Chinese Medicine
}

\author{
Zuyao Xie ${ }^{1}$, Gaiqin Yang2* \\ 1Shaanxi University of Traditional Chinese Medicine, Xianyang 712046, Shaanxi Province, China \\ ${ }^{2}$ Shaanxi Provincial Hospital of Traditional Chinese Medicine, Xi'an 710003, Shaanxi Province, China \\ *Corresponding author: Gaiqin Yang, yanggq-01@163.com
}

\begin{abstract}
Chronic obstructive pulmonary disease is abbreviated as "COPD," and its clinical incidence is more common. It is mostly based on a long-term treatment with western medicine, which has relatively large side effects and poor durability. At present, the curative effect of traditional Chinese medicine (TCM) treatment is obvious. Now a case of chronic obstructive pulmonary disease is introduced as follows.
\end{abstract}

Keywords: Chronic obstructive pulmonary; Acupoint embedding; Proven case

Publication date: July 2021; Online publication: July 31, 2021

\section{Patient introduction and main complaint}

The patient, male, 67 years old, was diagnosed for the first time on July 30, 2019. Main complaint was repeated cough and sputum for 3 years, worsening for 1 week. The patient had a cough and sputum after catching a cold 3 years ago, and there was no blood in the sputum. Symptoms improved after symptomatic treatment at the local hospital. Six months ago, he had cough again, a small amount of white sputum, difficult to cough up, no bloodshot eyes in the sputum, chest tightness, shortness of breath, wheezing, occasional dizziness, no hemoptysis, fever, night sweats, etc. He went to a nearby hospital for examination.

\section{CT of his chest}

CT of his chest showed that a few fibrous foci in the upper right lung; a few exudates and small nodules in the middle and lower lobes of the right lung, which improved after symptomatic treatment. One week ago, the patient recurred cough and sputum, accompanied by chest tightness, shortness of breath, wheezing, and dizziness. He wanted to seek TCM treatment, so he went to the instructor to see a doctor.

\section{Checking lung function}

When checking the lung function, it was observed that there was a mild obstructive pulmonary ventilation dysfunction, which is a small airway airflow that is mild to severely obstructed. The maximum ventilation per minute was normal; the diffusion function was basically normal, and the residual ratio was basically normal; the bronchodilation test was negative, the FEV1 variation rate was $0.8 \%$, and the absolute value difference of FEV1 was $0.02 \mathrm{~L}$. He was admitted to the hospital with "chronic obstructive pulmonary disease." 


\section{Admission symptoms}

It included were cough, a small amount of white sticky sputum, unfavorable cough and vomiting, chest tightness, shortness of breath, and wheezing, which aggravate after activities, sometimes dizziness, general appetite, poor night rest, and two bowel movements. Clear mind, good spirit, dark red tongue, thin white coating, tortuous veins under tongue, and thin pulse.

\section{Physical examination}

Observed that he had mild barrel chest, regular breathing rhythm. Clear mind, good spirit, dark red tongue, thin white coating, tortuous veins under tongue, and thin pulse. Percussion of both lungs showed clear sounds, low breath sounds in both lungs, and no dry and wet rales. Nervous system examination (-).

\section{Diagnosis}

(1) Western medicine diagnosis: It was diagnosed as chronic obstructive pulmonary disease.

(2) TCM diagnosis: It was diagnosed as lung bloating disease, phlegm stasis obstructing the lung and Qi deficiency and kidney deficiency syndrome.

\section{Treatment}

The treatment is based on acupuncture combined with TCM, with the method of "resolving phlegm and removing blood stasis, purging the lung and relieving asthma, and invigorating the kidney and Qi." Western medicine inhaled salmeterol and ticasone powder to relieve asthma.

\subsection{Acupuncture}

Acupuncture is combined with "Gualouxiebai Banxia Tang" to regulate the lungs and kidneys. The main points are as follows, "Feishu, Dingchuan, Gaolingshu, Pishu, Shenshu, Fengmen, Geshu, Ganshu, Xinshu, Yuji, Taiyuan." Matching points, "Zusanli, Sanyinjiao, Taixi, Fenglong, Baihui, Fengchi, Shenmen." Method of acupuncture was to allow the patient to be placed in a prone position, exposed to the skin, and routinely disinfected. A $0.25 \mathrm{~mm} \times 40 \mathrm{~mm}$ milli-needle is used to puncture the Fengchi acupoint with the tip of the needle facing the tip of the nose. After getting Qi, connect the bilateral Fengchi, Feishu-Geshu, Gaolingshu-Shenshu, Zusanli-Sanyin to the electroacupuncture instrument, using continuous wave, frequency $40 \sim 80 \mathrm{~Hz}$, to the patient's tolerance, lasting $20 \mathrm{~min}$, one time/d, five day is a course of treatment, and rest for two days afterwards. The prescription of TCM was Polygala 30g, melon wilt 15g, magnet 30g first decoction, Fushen 30g, Qing Pinellia 9g, Schisandra 15g, Trichosanthes 30g, Radix Rehmanniae 24g, Salvia 20g, Roasted Licorice $10 \mathrm{~g}$. One dose/day, warm in the morning and evening. After two courses of treatment, the patient's cough, sputum, chest tightness, shortness of breath, wheezing, and dizziness were all improved compared to before. After discharge from the hospital, acupoint embedding was performed. Choose bilateral Feishu, Pishu, Shenshu, Ganshu, Geshu, Xinshu, Dingchuan, Fengmen, Zusanli, Sanyinjiao, and Fenglong to regulate the five internal organs and benefit the lungs and kidneys. One time/seven day, three times as a course of treatment. Rest for one week after one course of treatment. After three courses of treatment, the above symptoms were significantly reduced compared with before. After three months of follow-up, the patient complained of occasional cough and sputum, the frequency of attacks was significantly reduced, chest tightness, shortness of breath, asthma did not worsen, dizziness disappeared, sleepiness was acceptable, and the second stool was adjusted.

\section{COPD complications, theories, countermeasures, future intervention}

Modern medicine believes that there are many complications of COPD, and the degree of danger increases 
during acute exacerbations. It is particularly important to control the number of acute exacerbations and alleviate the symptoms of this disease.

The mortality rate is relatively high, which has caused a serious economic burden to the society and the family ${ }^{[1,2]}$. Western medicine has high-cost treatment, high side effects, and damage to the viscera. The treatment of this disease by TCM stimulates the immune system as a whole, with few side effects and easy acceptance. As early as the "Huang Di Nei Jing," there is an explanation about lung diseases. "Suwen • Cough Theory" contains, "The five internal organs and six internal organs make people cough, not only the lung" points out that each internal organ can cause cough ${ }^{[3]}$. In "Treatise on Febrile and Miscellaneous Diseases," there are classifications of cough and asthma, Qi inversion, pulmonary dysfunction, and pulmonary carbuncle, reflecting the different stages of the disease. This disease belongs to the category of "lung bulging disease" in TCM. It is mostly caused by external infection invasion, improper diet, chronic illness and deficiency of Qi. The pathogenesis is deficiency of lung and kidney, internal resistance of phlegm and blood stasis. The disease is located in the lung, involving the liver, spleen and kidney. Take "Backshu: points including "Feishu, Xinshu, Pishu, Shenshu and Ganshu" is to regulate the balance of viscera. The blood will meet Geshu, the mastery of promoting blood circulation and removing blood stasis, asthma, replenishing the lung and relieving asthma, "yinlingquan," "Fenglong," expelling phlegm and removing blood stasis. Due to Qi deficiency and kidney deficiency, "zusanli and Sanyin" are used to harmonize Qi and blood. The above acupoints are treated with catgut embedding at acupoints to achieve long-term stimulation. In the decoction of TCM, "Gualou” clears away heat, clears up phlegm, widens the chest and clears up phlegm in the lungs. Pinellia dampness reduces phlegm. Polygala, Fushen, Magnet to soothe the nerves and nootropic. Fructus schisandra condenses the lungs to relieve cough. Trichosanthes, Rehmannia glutinosa nourishes yin and nourishes the lungs. Licorice reconciles various medicines. The whole viscera is harmonized, and the curative effect is considerable.

Acupoint embedding integrates a variety of therapies, and its long-lasting stimulating effect can enhance immunity. Catgut embedding at acupoints can control airway inflammation to a certain extent, reduce the release of inflammatory factors, and thereby inhibit the progression of lung disease ${ }^{[4]}$. Acupoint catgut embedding has a significant curative effect in the treatment of chronic obstructive pulmonary disease, which can reduce the pain of the number of visits for patients, and the long-term effect is considerable. At present, acupoint embedding can be used to treat a variety of clinical diseases, and has achieved good results, which is worthy of further research and promotion.

\section{Disclosure statement}

The author declares no conflict of interest.

\section{References}

[1] Liu Y, 2020, Clinical Analysis of Chronic Obstructive Pulmonary Disease Combined with Respiratory Failure Combined with Non-Invasive Positive Pressure Ventilation. Forum on Primary Medicine, 24(19): 2714-2716.

[2] Zhang ZL, Kang XW, Liang YL, et al., 2019, Analysis of "The Five Internal Organs and Six Internal Organs are Coughing, not the Lungs." Chinese Journal of Information on Traditional Chinese Medicine, 26(10): 124-126.

[3] Zhang D, Han X, Qin S, 2020, Clinical Observation of Acupoint Catgut Embedding in the Prevention and Treatment of Adverse Reactions after Chemotherapy in Advanced Non-Small Cell Lung Cancer. Shanghai Journal of Acupuncture, 39(9): 1113-1117. 